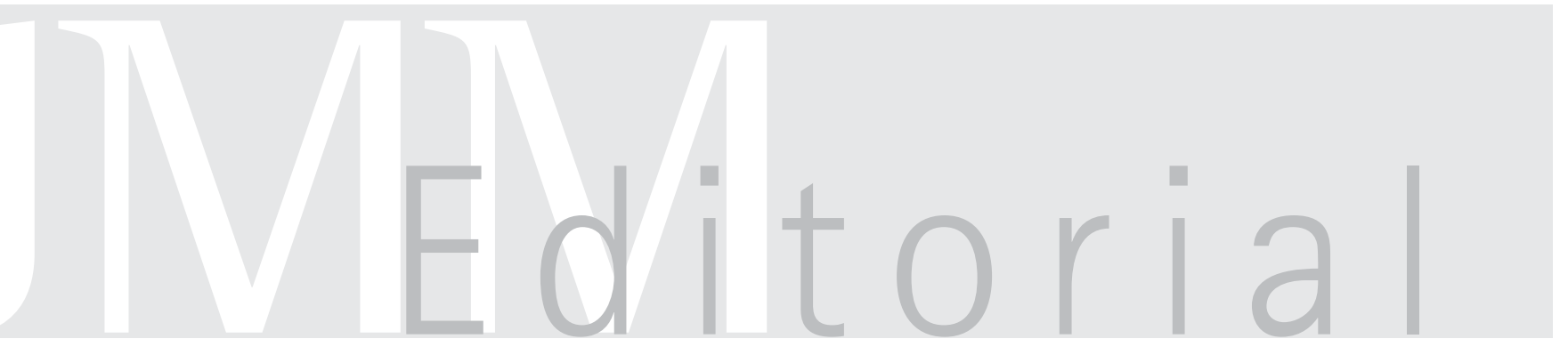

\title{
Chlamydial vaccine development
}

\section{Chlamydial infections}

Chlamydial organisms cause a wide spectrum of diseases in humans, other mammals and birds and have an enormous economic impact on both human and animal health and on agricultural industries worldwide. The two principal pathogens of humans are Chlamydia trachomatis and Chlamydophila pneumoniae (Schachter, 1988; Saikku, 1998). Chlamydia trachomatis is a cause of chronic conjunctivitis and is also the most common cause of sexually transmitted disease in humans. Infections can go unnoticed and, if left untreated, can ultimately result in scarring and fibrosis of ocular and genital tissues, resulting in trachoma (the leading cause of preventable blindness worldwide) and pelvic inflammatory disease, which can lead to infertility, ectopic pregnancy and chronic pelvic pain. Chlamydophila pneumoniae causes acute respiratory disease and is responsible for $5-10 \%$ of the cases of community-acquired pneumonia, bronchitis and sinusitis. The organism has also been associated with chronic obstructive pulmonary disease, asthma, reactive airway disease, Reiter's syndrome, sarcoidosis and atherosclerosis.

The principal pathogens of animals are Chlamydophila psittaci, Chlamydophila abortus, Chlamydophila pecorum, Chlamydia suis and Chlamydophila felis (Storz, 1988; Longbottom \& Coulter, 2003). Chlamydophila psittaci infects a wide variety of both wild and domesticated birds, including psittacine birds, such as parrots and budgerigars, where the disease is known as psittacosis, and game, pigeons and poultry, where the disease is known as ornithosis. Chlamydophila abortus is a major cause of abortion in sheep (ovine enzootic abortion or OEA) and goats. Cattle, pigs and horses can also be affected, but to a much lesser extent. Chlamydophila pecorum also affects ruminants and pigs, causing a wide range of conditions, including conjunctivitis, pneumonia, polyarthritis (stiff lamb disease), inapparent intestinal infections, mastitis, metritis and encephalomyelitis. In pigs, Chlamydia suis is also responsible for causing conjunctivitis, enteritis and pneumonia. Chlamydophila felis is a common cause of acute and chronic conjunctivitis in cats, particularly kittens. Two other animal pathogens, Chlamydia muridarum and Chlamydophila caviae, which respectively infect mice and guinea pigs, are important animal models for human chlamydial genital tract infections. Some of the animal pathogens are known to cause zoonotic infections in humans, particularly Chlamydophila psittaci and Chlamydophila abortus, and so are also of comparative medical interest.

\section{Is vaccination necessary?}

Chlamydial infections of both humans and animals can be treated effectively with antibiotics, although, once infection and pathology are established, treatment may be less effective. In humans, infection in many individuals is asymptomatic and so treating only those with clinical symptoms is not the best way of controlling the spread of infection (Stagg, 1998). One way of identifying asymptomatic individuals would be through a national screening programme, but, although this approach may be practicable for developed countries, it is likely to be too costly for developing countries. A vaccination programme would probably be much cheaper and have a greater impact on the prevalence of Chlamydia trachomatis infection. In terms of animal infections, again there are problems associated with asymptomatic infections. Furthermore, in OEA, for instance, antibiotic treatment is not guaranteed to prevent abortion or stop the shedding of infectious organisms at lambing or oestrus, which can then infect other animals (Longbottom \& Coulter, 2003). A combination of flock management, screening and vaccination is needed to control such infections.

\section{Vaccine development}

In order to develop effective vaccines for controlling and preventing chlamydial infections, it is important to understand the complex immunological mechanisms that occur during infection, to identify those antigens that elicit a protective immune response and also to develop effective methods for vaccine delivery. This brief overview summarizes the progress that has been made in these areas over the last 50 years and the future prospects for developing effective anti-chlamydial vaccines.

\section{Immunization with whole organisms}

Early animal and human vaccine studies in the 1950s and 1960s centred on the use of inactivated or live whole organisms (reviewed by Rank, 1999). However, in general, these vaccines have been more successful in controlling animal infections than human infections. In the veterinary field, highly effective live attenuated (a chemically induced temperature-sensitive mutant strain) and inactivated wholeorganism vaccines against OEA and inactivated vaccines against feline chlamydial infections are available commercially. The vaccines also reduce the shedding of infective organisms significantly and so limit the spread of infection. In humans, although short-term protection against ocular infection has been achieved with whole-organism vaccines, re-infection exacerbated disease in some individuals, suggesting that much of the pathological damage that occurs during chronic infections is caused by the host response. Following these studies, the use of whole organisms for developing human vaccines was essentially abandoned. However, once a stable genetic system for transformation of chlamydiae has been developed, it may be possible to produce a live attenuated vaccine, assuming that the causes of the immunopathological damage can be overcome.

\section{Immunization with chlamydial membrane components}

Following the identification of MOMP (major outer-membrane protein) as the major protein component (approx. 60\%) of the outer membrane in the early 1980s and the discovery that strong antibody responses are raised to the protein in infected animals and humans, vaccine studies focused on this protein. 
Experiments with denatured MOMP purified from SDS-PAGE gels, native MOMP purified as the major component of chlamydial outer-membrane complex preparations (COMCs) and passive immunization studies with mAbs to MOMP demonstrated the importance of conformational epitopes and neutralizing antibodies in controlling infection (reviewed by Rank, 1999). Control is probably achieved through limiting initial infection at a mucosal surface and through reducing the shedding and transmission of infective organisms.

\section{Immunization with recombinant MOMP}

In the early 1990s, attention turned to the development of recombinant protein vaccines, which would be easier and cheaper to prepare than either the whole-organism or COMC vaccines, which require the largescale production of purified chlamydiae, and would avoid all of the potential safety problems associated with live or oiladjuvanted whole-organism vaccines. However, this approach proved difficult, because the expression of full-length MOMP in prokaryotic expression systems is generally toxic, even at low levels. It is also difficult to produce recombinant MOMP in a native form with intact, conformationally relevant epitopes on a large enough scale to make it a viable option for use in a commercial vaccine. Some protection was achieved with various forms of recombinant MOMP, although efficacies were variable and not as good as those achieved with whole-organism vaccines (Herring et al., 1998), suggesting not only that conformation was important but that antigens other than MOMP may also be required.

\section{Protective immunity}

Following the recognition that the T cellderived cytokines IFN- $\gamma$ and TNF- $\alpha$ are important for the resolution of chlamydial infection and the identification of protective T-cell epitopes in MOMP, the importance of cell-mediated immune responses rather than neutralizing antibody became apparent in the mid 1990s. Adoptive transfer studies with chlamydia-specific Tcell clones and gene-knockout (particularly MHC class II, T-cell receptor $\alpha \beta$ and IFN- $\gamma$ ) mouse studies have shown that an MHC class II-restricted, $\mathrm{CD} 4^{+}$T-helper type 1 (Th1) response, mediated by IFN- $\gamma$ and TNF- $\alpha$ production, is critical for resolving primary infection (reviewed by Rank, 1999; Ward, 1999; Igietseme, 2002). Dendritic cells have also been shown to play an important role in anti-chlamydial immunity, as mice immunized with dendritic cells pulsed ex vivo with chlamydial antigen develop a Th1dominant response and produce large amounts of the cytokine IL-12, which stimulates IFN- $\gamma$ production (Lu \& Zhong, 1999). MHC class I-restricted cytotoxic $\mathrm{CD}^{+} \mathrm{T}$ cells may also be involved in resistance to infection, but this appears to be through IFN- $\gamma$ production rather than cytolytic function (Buzoni-Gatel et al., 1992; Lampe et al., 1998b).

In contrast to cellular immune responses, the relative contribution of antibody to resolving infection is still a matter of debate. Studies in the guinea pig model for human genital tract infections have demonstrated a role for antibody in limiting primary infection (Rank et al., 1979; Rank \& Batteiger, 1989), whereas B cell-deficient gene-knockout studies have shown that there appears to be no requirement for antibody in the murine model, although these mice are more susceptible to reinfection than are immunocompetent mice (Su et al., 1997). Studies in Fc receptorknockout mice (Moore et al., 2002) suggest that the predominant role of antibodies is in resistance to re-infection, by enhancing the uptake, processing and presentation of chlamydial antigens by antigen-presenting cells for rapid Th1 activation and clearance of infection.

\section{Identifying other protective antigens}

Although MOMP has been the main target antigen for vaccine development over the last 20 years, other chlamydial components may also play a role in protection. In our laboratory, we have been particularly interested in the polymorphic (outer-) membrane proteins (POMPs or Pmps), which were identified as minor but immunodominant components of the protective COMC preparation

(Longbottom et al., 1998b). Genomic sequencing of Chlamydia trachomatis and Chlamydophila pneumoniae identified nine and 21 orthologous genes, respectively (Stephens et al., 1998; Read et al., 2000), while preliminary analysis of the recently completed Chlamydophila abortus genome has identified at least a further 12

paralogous genes in addition to the original six (J. Parkhill, D. Longbottom and others, unpublished), revealing the complexity of this group of proteins. The POMPs, some of which have been shown to be surfaceexposed (Longbottom et al., 1998a), share similarities with type- $\mathrm{V}$ autotransporters (Henderson \& Lam, 2001) and one may function as a cytolysin (Lampe et al., 1998a). They have been suggested to play an important role in chlamydial host immune evasion, using recombination, strandslippage or differential expression as possible mechanisms of antigenic variability. The fact that such a large proportion of the small chlamydial genome encodes this family of proteins suggests that host immune evasion is of prime importance for these organisms and that they are important vaccine candidate antigens. Indeed, the TRACVAX recombinant subunit vaccine from Antex Biologics, which will be assessed in phase 1 human clinical trials in 2003 (Antex Biologics, 2003), is based on the Chlamydia trachomatis 'superfamily' of nine POMPs. This will be the first human vaccine trial against Chlamydia trachomatis for nearly 40 years and represents an important milestone in human chlamydial vaccine development.

Other proteins of interest are the 60 and $75 \mathrm{kDa}$ heat-shock proteins, the type-III secretory system structural proteins (Hsia et al., 1997), exoglycolipid (Stuart et al., 1987) and those that have access to the hostcell cytoplasm and therefore may be presented by the MHC class I pathway to $\mathrm{CD}^{+}{ }^{+} \mathrm{T}$ cells, such as the type-III secretory system effector proteins. Such proteins could include the Inc proteins (Rockey et al., 2000) and Cap1 (Fling et al., 2001), which are localized to the inclusion membrane. In addition, many other outer-membrane surface components that have been predicted following genome sequencing and proteomic analysis await investigation.

\section{New approaches to chlamydial vaccine development}

Over the last 10 years, a number of new approaches have been taken to the identification of protective antigens other than MOMP that can be used in a subunit vaccine and to the development of effective methods for vaccine delivery that elicit systemic and mucosal immune responses, particularly sustained mucosal responses against Chlamydia trachomatis infections (reviewed by Igietseme, 2002).

The use of microbial delivery systems such as attenuated Salmonella typhimurium 
(Hayes et al., 1991), a hybrid poliovirus (Murdin et al., 1993), a novel potato virus X plant expression system (Herring et al., 1998) and Lactobacillus fermentum (Turner \& Giffard, 1999), present as part of the normal vaginal flora, have shown some promise. Other bacterial delivery systems such as virulence-attenuated strains of Salmonella, Vibrio cholerae and Listeria monocytogenes (Gentschev et al., 2002) and viral vectors such as non-infectious adenovirus, canarypox virus and vaccinia virus could also be investigated (Igietseme, 2002). More recently, genetic or DNA vaccination has been explored as a means of inducing protective immunity in a variety of animal model systems (Entrican et al., 2001). However, this approach has been more successful in eliciting protective Th1 responses in the mouse model for human respiratory infections (Zhang et al., 1997), rather than for genital tract infections (Pal et al., 1999). DNA vaccination has also been effective in protecting turkeys from avian chlamydiosis (Vanrompay et al., 1999), but has so far been less effective in protecting sheep from Chlamydophila abortus infection (L. Coulter, D. Longbottom and others, unpublished data). The reasons for the variability in success are unclear, but are probably a consequence of different immunization protocols and a reflection of the different protective mechanisms required for the different infections. Nevertheless, DNA vaccination represents an attractive method for delivery of subunit vaccines and should be explored further as a means of enhancing immunogenicity and eliciting protective immunity. Factors that could be considered to improve vaccine efficacy include codon optimization, the delivery vehicle, the route of immunization, a prime-boost vaccination strategy and the inclusion of co-stimulatory molecules, such as cytokines and chemokines, and CpG immunostimulatory sequences that enhance Th1 immune responses.

Hormones associated with the oestrus cycle and pregnancy may also have a significant effect on the genital mucosal immune response to vaccination against Chlamydia trachomatis and the systemic response to vaccination against Chlamydophila abortus.

DNA vaccination has also been used successfully as a tool for screening selected ORFs from the Chlamydophila pneumoniae genome to identify new potential vaccine candidates (Murdin et al., 2000). Using this approach, the $60 \mathrm{kDa}$ cysteine-rich protein and an ADP/ATP translocase were identified in addition to MOMP as protective antigens. The use of bonemarrow-derived mouse dendritic cells pulsed ex vivo with chlamydial antigen (Su et al., 1998) provides another way of identifying antigens that elicit a Chlamydiaspecific, IL-12-dependent, CD4 ${ }^{+}$Th 1 immune response in vitro, although this is not necessarily predictive of the immune response generated in vivo (Shaw et al., 2002).

\section{Future vaccine perspective}

Vaccination remains the best approach for protection of the largest number of people and animals from chlamydial infections and their complications worldwide. The generation of a long-lasting, protective immune response will probably be best achieved with the development of a multicomponent recombinant subunit vaccine. Therefore, the ultimate goal of chlamydial vaccine research is to identify protective antigens, as well as safe and effective vaccine delivery methods that are capable of inducing the necessary protective immune response. Central to this goal is the need to develop safe delivery vehicles and adjuvants for optimal antigen presentation. Novel methods are also now available, aided by the increasing availability of sequenced chlamydial genomes, for identification of antigens that could ultimately be incorporated in a subunit vaccine (Su et al., 1998; Murdin et al., 2000), avoiding the components responsible for mediating immunopathological damage. Ideally, any vaccine should elicit sterilizing immunity and, in the case of human infections, it should generate and sustain an adequate protective immune response at the mucosal surface and also offer cross-protection against the different serovars of Chlamydia trachomatis. Interestingly, computer modelling has suggested that even a partially efficacious vaccine would lead to a rapid decrease in the prevalence of Chlamydia trachomatis genital tract infection in the population (de la Maza \& de la Maza, 1995) and so may be an acceptable short-term objective. Similarly, for animal infections, it would be important that vaccines protect against the different subtypes/serovars of Chlamydophila pecorum and Chlamydophila psittaci and also against the increasing number of isolated variant strains of Chlamydophila abortus. Importantly, they should also limit the shedding of infectious organisms to prevent the transmission of infection and limit possible zoonotic transmission to humans. Vaccine studies to date would suggest that an efficacious antichlamydial vaccine should induce not only strong mucosal and systemic Th1 immune responses, but also a humoral response that enhances Th1 activation following a secondary infection. It is therefore encouraging to see that protective efficacy results from animals immunized mucosally with the TRACVAX vaccine show that it protects against both upper and lower reproductive tract Chlamydia trachomatis infection and confers heterotypic crossprotection against the most common strains associated with urogenital tract infections and that protection correlates with both cellular and humoral immune responses. Therefore, overall, there is every reason to be optimistic about the prospects for developing safe and effective vaccines to control the wide range of chlamydial infections affecting both humans and animals.

\section{David Longbottom}

Moredun Research Institute, Pentlands Science Park, Bush Loan, Penicuik, Midlothian EH26 OPZ, UK

\section{Correspondence: David Longbottom (longd@mri.sari.ac.uk)}

Antex Biologics (2003). Antex files Chlamydia investigational new drug application with FDA. STD vaccine clinical trial. Press release, 3

February 2003. http://

www.antexbiologics.com/news_pr/ pr030203.phtm

Buzoni-Gatel, D., Guilloteau, L., Bernard, F., Bernard, S., Chardes, T. \& Rocca, A. (1992). Protection against Chlamydia psittaci in mice conferred by Lyt- $2^{+} \mathrm{T}$ cells. Immunology 77 , 284-288.

de la Maza, M. A. \& de la Maza, L. M. (1995). A new computer model for estimating the impact of vaccination protocols and its application to the study of Chlamydia trachomatis genital infections. Vaccine 13, 119-127.

Entrican, G., Buxton, D. \& Longbottom, D. (2001). Chlamydial infection in sheep: immune control versus fetal pathology. J R Soc Med 94, 273-277.

Fling, S. P., Sutherland, R. A., Steele, L. N., Hess, B., D'Orazio, S. E. F., Maisonneuve, J., Lampe, M. F., Probst, P. \& Starnbach, M. N. (2001). CD8 ${ }^{+} \mathrm{T}$ cells recognize an inclusion membrane-associated protein from the vacuolar pathogen Chlamydia trachomatis. Proc Natl Acad Sci U S A 98, 1160-1165.

Gentschev, I., Dietrich, G., Spreng, S., Pilgrim, S., Stritzker, J., Kolb-Maurer, A. \& Goebel, W. 
(2002). Delivery of protein antigens and DNA by attenuated intracellular bacteria. Int $\mathrm{J} \mathrm{Med}$ Microbiol 291, 577-582.

Hayes, L. J., Conlan, J. W., Everson, J. S., Ward, M. E. \& Clarke, I. N. (1991). Chlamydia trachomatis major outer membrane protein epitopes expressed as fusions with LamB in an attenuated aroA strain of Salmonella typhimurium; their application as potential immunogens. J Gen Microbiol 137, 1557-1564.

Henderson, I. R. \& Lam, A. C. (2001).

Polymorphic proteins of Chlamydia spp. autotransporters beyond the Proteobacteria. Trends Microbiol 9, 573-578.

Herring, A. J., Jones, G. E., Dunbar, S. M., Nettleton, P. F., Fitzgerald, T. A., Anderson, I. E., Chapman, S. N. \& Wilson, T. M. A. (1998). Recombinant vaccines against Chlamydia psittaci -an overview of results using bacterial expression and a new approach using a plant virus 'overcoat' system. In Proceedings of the Ninth International Symposium on Human Chlamydial Infection, pp. 434-437. Edited by R. S. Stephens, G. I. Byrne, G. Christiansen \& 7 others. San Francisco: International Chlamydia Symposium.

Hsia, R. C., Pannekoek, Y., Ingerowski, E. \& Bavoil, P. M. (1997). Type III secretion genes identify a putative virulence locus of Chlamydia. Mol Microbiol 25, 351-359.

Igietseme, J. U. (2002). Functional immunobiology of Chlamydia: vaccine perspective. In Proceedings of the Tenth International Symposium on Human Chlamydial Infections, pp. 223-232. Edited by J. Schachter, G. Christiansen, I. N. Clarke \& 11 others. San Francisco: International Chlamydia Symposium.

Lampe, M. F., Ballweber, L. M. \& Stamm, W. E. (1998a). Cloning and sequence analysis of a putative cytolysin from Chlamydia trachomatis. In Proceedings of the Ninth International Symposium on Human Chlamydial Infection, pp. 555-558. Edited by R. S. Stephens, G. I. Byrne, G. Christiansen \& 7 others. San Francisco: International Chlamydia Symposium.

Lampe, M. F., Wilson, C. B., Bevan, M. J. \& Starnbach, M. N. (1998b). Gamma interferon production by cytotoxic $\mathrm{T}$ lymphocytes is required for resolution of Chlamydia trachomatis infection. Infect Immun $\mathbf{6 6}$ 5457-5461.

Longbottom, D. \& Coulter, L. J. (2003). Animal chlamydioses and zoonotic implications. J Comp Pathol 128, 217-244.

Longbottom, D., Findlay, J., Vretou, E. \& Dunbar, S. M. (1998a). Immunoelectron microscopic localisation of the OMP90 family on the outer membrane surface of Chlamydia psittaci. FEMS Microbiol Lett 164, 111-117. Longbottom, D., Russell, M., Dunbar, S. M.,
Jones, G. E. \& Herring, A. J. (1998b).

Molecular cloning and characterization of the genes coding for the highly immunogenic cluster of 90-kilodalton envelope proteins from the Chlamydia psittaci subtype that causes abortion in sheep. Infect Immun 66, 1317-1324.

Lu, H. \& Zhong, G. M. (1999). Interleukin-12 production is required for chlamydial antigenpulsed dendritic cells to induce protection against live Chlamydia trachomatis infection. Infect Immun 67, 1763-1769.

Moore, T., Ananaba, G. A., Bolier, J., Bowers, S., Belay, T., Eko, F. O. \& Igietseme, J. U. (2002). Fc receptor regulation of protective immunity against Chlamydia trachomatis. Immunology 105, 213-221.

Murdin, A. D., Su, H., Manning, D. S., Klein, M. H., Parnell, M. J. \& Caldwell, H. D. (1993). A poliovirus hybrid expressing a neutralization epitope from the major outer membrane protein of Chlamydia trachomatis is highly immunogenic. Infect Immun 61, 4406-4414.

Murdin, A. D., Dunn, P., Sodoyer, R., Wang, J., Caterini, J., Brunham, R. C., Aujame, L. \& Oomen, R. (2000). Use of a mouse lung challenge model to identify antigens protective against Chlamydia pneumoniae lung infection. J Infect Dis 181 (Suppl. 3), S544-S551.

Pal, S., Barnhart, K. M., Wei, Q., Abai, A. M., Peterson, E. M. \& de la Maza, L. M. (1999). Vaccination of mice with DNA plasmids coding for the Chlamydia trachomatis major outer membrane protein elicits an immune response but fails to protect against a genital challenge. Vaccine 17, 459-465.

Rank, R. G. (1999). Models of immunity. In Chlamydia: Intracellular Biology, Pathogenesis and Immunity, pp. 239-295. Edited by R. S. Stephens. Washington, DC: American Society for Microbiology.

Rank, R. G. \& Batteiger, B. E. (1989).

Protective role of serum antibody in immunity to chlamydial genital infection. Infect Immun 57, 299-301.

Rank, R. G., White, H. J. \& Barron, A. L. (1979). Humoral immunity in the resolution of genital infection in female guinea pigs infected with the agent of guinea pig inclusion conjunctivitis. Infect Immun 26, 573-579.

Read, T. D., Brunham, R. C., Shen, C. \& 22 other authors (2000). Genome sequences of Chlamydia trachomatis MoPn and Chlamydia pneumoniae AR39. Nucleic Acids Res 28 1397-1406.

Rockey, D. D., Lenart, J. \& Stephens, R. S. (2000). Genome sequencing and our understanding of chlamydiae. Infect Immun 68, 5473-5479.

Saikku, P. (1998). Chlamydia pneumoniae clinical spectrum. In Proceedings of the Ninth International Symposium on Human
Chlamydial Infection, pp. 145-154. Edited by R. S. Stephens, G. I. Byrne, G. Christiansen \& 7 others. San Francisco: International Chlamydia Symposium.

Schachter, J. (1988). Overview of human diseases. In Microbiology of Chlamydia, pp. 153-165. Edited by A. L. Barron. Boca Raton, FL: CRC Press

Shaw, J., Grund, V., Durling, L., Crane, D. \& Caldwell, H. D. (2002). Dendritic cells pulsed with a recombinant chlamydial major outer membrane protein antigen elicit a $\mathrm{CD} 4^{+}$type 2 rather than type 1 immune response that is not protective. Infect Immun 70, 1097-1105.

Stagg, A. J. (1998). Vaccines against Chlamydia: approaches and progress. Mol Med Today 4, 166-173.

Stephens, R. S., Kalman, S., Lammel, C. \& 9 other authors (1998). Genome sequence of an obligate intracellular pathogen of humans: Chlamydia trachomatis. Science 282, 754-759.

Storz, J. (1988). Overview of animal diseases induced by chlamydial infections. In Microbiology of Chlamydia, pp. 167-192. Edited by A. L. Barron. Boca Raton, FL: CRC Press.

Stuart, E. S., Tirrell, S. M. \& MacDonald, A. B. (1987). Characterization of an antigen secreted by Chlamydia-infected cell culture. Immunology 61, 527-533.

Su, H., Feilzer, K., Caldwell, H. D. \& Morrison, R. P. (1997). Chlamydia trachomatis genital tract infection of antibody-deficient gene knockout mice. Infect Immun 65, 1993-1999.

Su, H., Messer, R., Whitmire, W., Fischer, E., Portis, J. C. \& Caldwell, H. D. (1998).

Vaccination against chlamydial genital tract infection after immunization with dendritic cells pulsed ex vivo with nonviable chlamydiae. J Exp Med 188, 809-818.

Turner, M. S. \& Giffard, P. M. (1999)

Expression of Chlamydia psittaci- and human immunodeficiency virus-derived antigens on the cell surface of Lactobacillus fermentum BR11 as fusions to BspA. Infect Immun 67, 5486-5489.

Vanrompay, D., Cox, E., Vandenbussche, F., Volckaert, G. \& Goddeeris, B. (1999).

Protection of turkeys against Chlamydia psittaci challenge by gene gun-based DNA immunizations. Vaccine 17, 2628-2635.

Ward, M. E. (1999). Mechanisms of disease. In Chlamydia: Intracellular Biology, Pathogenesis and Immunity, pp. 171-210. Edited by R. S.

Stephens. Washington, DC: American Society for Microbiology.

Zhang, D., Yang, X., Berry, J., Shen, C., McClarty, G. \& Brunham, R. C. (1997). DNA vaccination with the major outer-membrane protein gene induces acquired immunity to Chlamydia trachomatis (mouse pneumonitis) infection. J Infect Dis 176, 1035-1040. 